\title{
Exosome-Mediated siRNA pool Inhibits Axonal Growth Inhibitor in Cortical Neurons of Spinal Cord Injury in Rats
}

Qi Liao (D qiliao_20200526@sina.com )

Wuhan University Renmin Hospital https://orcid.org/0000-0002-7524-8954

Jiang-Hua Ming

Wuhan University Renmin Hospital

Ge-Liang Hu

Wuhan University Renmin Hospital

Research article

Keywords: SCl, exosome axonal growth inhibitor, Troy, PTEN, NgR, LINGO-1

Posted Date: July 14th, 2020

DOI: https://doi.org/10.21203/rs.3.rs-41026/v1

License: (c) (i) This work is licensed under a Creative Commons Attribution 4.0 International License.

Read Full License 


\section{Abstract}

Background: As exosomes have been confirmed as a reservoir of siRNAs involved in certain diseases, the current study aims to investigate whether exosomal-siRNA could exert a protective role in spinal cord injury (SCI).

Methods and Results: Exosomes in our experiment were isolated from lysosomal membrane-associated protein 2b (Lamp2b) overexpression HEK 293T cells, and purity of exosomes was characterized by the expression of $C D 9, C D 47$, and CD63 via western blot. Furthermore, the siRNA pool contains four siRNAs including siRNA-NgR, siRNA-LINGO-1, siRNA-Troy, and siRNA-PTEN was loaded to the exosomes, which indicated a significant role for the siRNA pool in reducing the expression of axon growth inhibitory factors. Upon the completion of loading into exosomes (exo-siRNA pool), the exo-siRNA pool was injected into primary cortical neurons of the $\mathrm{SCl}$ model in rats before cell proliferation and Rho expression were determined With the results revealed that purified addition could be applied to future experiments. The exo-siRNA pooled transfection caused downregulation of axon growth suppressors in primary cortical neurons including Nogo receptors $(\mathrm{NgR})$, leucine-rich repeats and immunoglobulin domain-containing protein 1 (LINGO-1), Troy, and phosphatase and tenson homolog (PTEN). Cell proliferation and Rho expression of primary cortical neurons inhibited the expression of axonal growth inhibitors in rats with $\mathrm{SCl}$ by transfecting exogenous Sirna.

Conclusion: This study confirmed that exosomes derived from Lamp2b overexpression HEK 293T cells facilitated both the recovery of functions and the survival of neurons when being loaded with the siRNA pool.

\section{Introduction}

$\mathrm{SCl}$ is regarded as one of the most serious diseases in spine injury, which frequently leads to impaired motor function. Causes of $\mathrm{SCl}$ comprise of traffic accidents, falls, and violent injuries.[1] Despite the advancement of medical technologies contributes a lot to the survival rate of $\mathrm{SCl}$ patients, the persistent impairment in central functions remains unchanged due to the lack of effective therapeutic options for patients. It's essential to explore effective therapeutic measures for the rehabilitation and regeneration of the injured spinal cord. Recent reports have demonstrated that the process of $\mathrm{SCl}$ recovery can be affected at the absence of pro-nerve growth factors and the presence of axonal growth inhibitors, both of which hinder the recovery of SCl.[2] Be it the structure or function of cortical neurons, the two causes are of vital importance for the corticospinal tracts. It has been reported the interference of axonal growth inhibitors may function as potential targets for rehabilitation and regeneration of the damaged corticospinal tracts.[3]

Involved in diverse physiological and pathological processes,[4] exosomes are membrane vesicles with diameters of 40-100 $\mathrm{nm}$ secreted by various cells and delivered into the extracellular micro-environment by different types of cells.[5] Today, exosomes have emerged as promising nanocarriers in drug 
administration, via carrying proteins, lipids, and genetic materials that reflect the origin of cells, facilitating intercellular communication and inducing multiple biological effects locally or distally. Small interfering RNA (siRNA) is a molecule of RNA which is carried by viruses into cells and has been employed as a technique to silence the expression of the gene of interests. Besides, siRNA also could be translocated into cells or tissues by exosomes encapsulation for the accomplishment of knockdown target genes. The treatment of numerous diseases in recent years has taken advantage of exosomes loaded with siRNA including autism,[6] stroke[7], and traumatic brain injury.[8]

Over the past two decades, multiple axonal growth inhibitors located in the central nervous system (CNS) have been proved by researches that these factors aggravate the damage to the axon, such as Nogo transmembrane proteins,[9] myelin associated glycoproteins,[10] odendrocyte myelin glycoproteins[11] as well as glia scar[12]. The presence of axon growth inhibitors presents an explanation of why axons of the central nervous system are incapable of regeneration after injury. Moreover, knocking out the expression of axon growth inhibitors using RNA interference may exert protective effects on SCI.[13] As a critical regulator of tumorigenesis and bone remodeling, PTEN also presents in periodontal tissues. A dramatic decrease of PTEN has displayed in the ligature-induced mouse periodontitis model which is of great significance in periodontitis and bone remodeling.[14] Furthermore, low expression of PTEN features as one of the characteristics of cranial meningiomas.[15] Serving as a transmembrane protein, LINGO-1 negatively regulates neural regeneration in the CNS,[16], and the expression of which is up-regulated after central nerve injury accompanying cell death. Although the relationship between LINGO-1 and cell death has not been characterized, LINGO-1 deletion and positive microenvironment may produce synergistic effects on the promotion of nerve fiber regeneration.[17] Thus the inhibition of LINGO-1 may be applied as a therapeutic strategy to promote neural regeneration of $\mathrm{SCl}$. $\mathrm{NgR}$, the axon growth inhibitory factor also plays a critical role for the recovery of injured nerves in multiple sclerosis, which has been confirmed that chemicals involved in acute $\mathrm{SCl}$ regulate the expressions of Nogo-A and $\mathrm{NgR}$ and stimulate the regeneration of nerve cells.[18] TNFRSF is a member of the tumor necrosis factor receptor superfamily[19] which can be expressed on the mouse embryo (Troy), and synthesized as a complex of LINGO-1 and NgR. Both of them inhibit axon growth by mediating inhibitor signals. More importantly, PTEN functions as a gene regulator that participates in a cellular signaling network. Our proposal has been made on the previous reports to explore the effect of siRNA pool in axon growth inhibitors.

In our study, we constructed the Lamp2b-overexpression 293T cells for the isolation of exosomes. The siRNA pool was transfected to primary cortical neurons and we hypothesis that the cortical neuron functions can be strengthened by injecting the exosomes-siRNA pools.

\section{Materials And Methods}

Cell culture. HEK293 cells were purchased from the Cell Bank of the Chinese Academy of Sciences (Shanghai, China). The cells were cultured in Dulbecco's modified Eagle's medium (DMEM) supplemented with $10 \% \mathrm{FBS}$ and $1 \% \mathrm{P} / \mathrm{S}$ at $37{ }^{\circ} \mathrm{C}$ in a $5 \% \mathrm{CO}_{2}$ atmosphere. 
Primary cortical neurons in the $\mathrm{SCl}$ rat model. The $\mathrm{SCl}$ rat model was induced by extradural compression using Allen's method as previously described.[20] Sprague-Dawley rats were anesthetized by injecting $10 \%$ chloral hydrate $(3 \mathrm{~mL} / \mathrm{kg})$ intraperitoneally, with furs on the back shaved under an aseptic condition and the T9-11 spinous process and vertebral segments were exposed. Then compression was impacted on the T10 spinous process at an interval of $12.5 \mathrm{~mm}$ for $20 \mathrm{~s}$ to produce a severe injury. Paralysis of the lower limbs was defined as a successful SCI model. After surgeries, all animals were introduced penicillin and analgesics three days. Cortical neurons were collected from the $\mathrm{SCl}$ rat model, Sterilization was performed by immersing in $75 \%$ ethanol after cervical dislocation. Cortical tissues were dissected from the brains in DMEM (Invitrogen, Carlsbad, CA), dissociated mechanically, chopped gently 15 times, and digested with $0.25 \%$ trypsin (Invitrogen) at $37^{\circ} \mathrm{C}$ for 25 minutes. The digestion was terminated with DMEM containing $10 \%$ fetal bovine serum (FBS). Mixed gently and a single-cell suspension was obtained. The suspension was then filtered through nylon meshes, centrifuged at $500 \mathrm{~g}$ for $5 \mathrm{~min}$, resuspended in DMEM with $10 \% \mathrm{FBS}, 2 \mathrm{~g} / \mathrm{L}$ of HEPES, $100 \mathrm{U} / \mathrm{mL}$ of penicillin $\mathrm{G}$, and $100 \mu \mathrm{g} / \mathrm{mL}$ of streptomycin (Invitrogen, Carlsbad, CA) and plated at a density of $1 \times 10^{6} / \mathrm{mL}$ in poly-L-lysine-coated plates or coverslips. After being cultured 6 days, cells were ready for experiments. All experiments were initiated on day 7 after cell implantation and were replaced 1 day before transfection fluid.

Lentiviral vectors transduction. Following the manufacturer's instructions, the HEK 293T cells were seeded in six-well plates, transfected with $2 \mu \mathrm{g}$ of DNA (pcDNA3.1-RVG-Lamp2b or empty pcDNA3.1 plasmid) using $10 \mu \mathrm{L}$ of Lipofectamine 2000 reagent in $250-250 \mu \mathrm{L}$ of Opti-MEM. The mixture was incubated for $5 \mathrm{~min}$ and then co-incubated at room temperature for $20 \mathrm{~min}$. Serum and antibiotics-free DMEM cells were added to the complex. Our preliminary experiments revealed that the protein expression of the transfected $293 \mathrm{~T}$ cells was at the peak at $24 \mathrm{~h}$, which was maintained at both $24 \mathrm{~h}$ and $48 \mathrm{~h}$.

Exosomes isolation from HEK $293 \mathrm{~T}$ cells. The cells were cultured with $10 \%$ of exosome-depleted FBS at $37^{\circ} \mathrm{C}$ for two days. The cell suspension was harvested and exosomes were isolated by differential centrifugation $(2,000 \times \mathrm{g}$ for $10 \mathrm{~min}, 10,000 \times \mathrm{g}$ for $30 \mathrm{~min}, 100,000 \times \mathrm{g}$ for $60 \mathrm{~min}$, and $100,000 \times \mathrm{g}$ for $60 \mathrm{~min})$. Exosome pellets were resuspended in PBS.

Determination of exosomes isolated from 293T cells. We made a further identification of exosomes to detect the expression of specific CD9, CD47, and CD63 antibody subject to western blot.

Labeling of siRNA. The siRNA pool containing four siRNAs ( si-Tories, si-PTEN, si-LINGO-1, and si-NgR) was labeled with Cy 5 using a Label IT ${ }^{\circledR}$ siRNA Tracker Cy $5^{\text {TM }}$ Kit (Mirus Bio LLC, Madison, WI, Cat \#7213, red, excitation of 649 emission of 670 ) according to manufactures' instructions of use.

Loading of exosomes with siRNA. Exosomes were introduced with the siRNA pool by employing the ExoFect Exosome Transfection Kit (SBI, China). Of $50 \mu \mathrm{L}$ exosomes or PBS suspension ( $50 \mu \mathrm{g})$ were mixed with $10 \mu \mathrm{L}$ of Exo-Fect solution and $20 \mu \mathrm{L}$ of Cy5-labeled siRNA pool. Exosomes $(40 \mu \mathrm{L})$ were incubated with $0.1 \mathrm{nmol}$ of cy3-PTEN-siRNA, cy3-NgR-siRNA, cy3-LINGO-1-siRNA, and cy3-Troy-siRNA (Advirna, USA) 
in an Eppendorf at $37^{\circ} \mathrm{C}$ for $2-3 \mathrm{~h}$. The suspension was subjected to Nanosight analysis. The concentration ratio of the fluorescent vs nonfluorescent exosomes was defined as loading efficiency.

Transmigration of loaded exosomes in primary cortical neurons. The exosomes were loaded in the siRNA pool, placed together with cortical neurons, and incubated for $24 \mathrm{~h}$. To determine the transmigration of exosomes with siRNA, cells were photographed at the presence of siRNA (red, excitation of 585/29 emission of 628/32) using the Cell Imaging System. Fluorescent intensity was measured utilizing Image $\mathrm{J}(\mathrm{NIH})$ to quantify Texas-Red control siRNA and the transmigration.

Cell viability. MTT assay was performed to detect cell viability. The cortical neurons were seeded into 96well plates at a density of 5,000 cells per well. The cells of the control group were incubated in complete medium at $37^{\circ} \mathrm{C}$ for $24 \mathrm{~h}$. The remaining groups were replaced with complete medium containing exosiRNA pool in an incubator with $5 \% \mathrm{CO}_{2}$ at $37^{\circ} \mathrm{C}$ for $48 \mathrm{~h}$. The media was removed and cells were incubated in a $20 \mu \mathrm{L}$ of MTT for $4 \mathrm{~h}$, and then $150 \mu \mathrm{L}$ of DMSO was added to dissolve intracellular and form azan crystals for $10 \mathrm{~min}$. Optical density (OD) was detected with an Infinite F200 (TecanInc, Switzerland) at $490 \mathrm{~nm}$. Cell viability (\%) was calculated as the following formula: (OD treated - OD blank /OD control $) \times 100 \%$, where OD treated was the OD obtained at the presence of the samples, OD control was the absorbance of cells that were not treated with samples, and OD blank was the absorbance of serum-free medium.

RT-PCR was carried out to determine the mRNA levels of gene expression, total RNA was extracted by using Trizol reagent which was then reverse-transcribed into CDNA in a 20 volume applying a TAKARA reverse-transcriptase (RT)-PCR kit. Amplification was performed for 30 cycles at $94^{\circ} \mathrm{C}$ for $30 \mathrm{~s}, 60^{\circ} \mathrm{C}$ for $30 \mathrm{~s}, 72{ }^{\circ} \mathrm{C}$ for $2 \mathrm{~min} ; 25$ cycles at $94^{\circ} \mathrm{C}$ for $30 \mathrm{~s}, 58^{\circ} \mathrm{C}$ for $30 \mathrm{~s}, 72{ }^{\circ} \mathrm{C}$ for 2 minutes in a DNA thermal cycle heat block (Bio-Rad, CFX96). The primer pairs employed were as follows, and GAPDH normalized data were presented as multiple changes in gene expression.

Western blot. Total protein was extracted from the cortical neurons and the concentration was measured by BCA protein assay. The prepared proteins were separated with $6 \%$ sodium do decyl sulphate polyacrylamide gel electrophoresis and transferred to polyvinylidene fluoride (PVDF) membranes with $350 \mathrm{~mA}$ for $4 \mathrm{~h}$. Then, PVDF membranes were blocked with $5 \%$ skimmed milk after dissolved in tris buffered saline tween (TBST) at room temperature for $2 \mathrm{~h}$ and incubated with primary antibodies at $4{ }^{\circ} \mathrm{C}$ overnight. Next, PVDF membranes were incubated with horseradish peroxidase-conjugated anti-rabbit IgG secondary antibodies (dilution: 1/2000; Cat. no.: ab6721; Abcam, Cambridge, UK) at room temperature for $2 \mathrm{~h}$ and visualized using ECL chemiluminescence (Milipore, Waltham, USA) by a Molecular Imager ChemiDoc XRS System (Bio-Rad Laboratories, Hercules, CA). The antibodies were as follows: CD63 (dilution: 1/1000, Abcam, UK), CD47 (dilution: 1/1000; Cell Signaling Technology, MA), CD9 (dilution: 1/1000; Cell Signaling Technology, MA), Troy (dilution: 1/1000; Cell Signaling Technology, MA), PTEN (dilution: 1/500, Cell Signaling Technology, MA), LINGO-1 (dilution: 1/500, Cell Signaling Technology, MA) and NgR (dilution: 1/500; Cell Signaling Technology, MA). Band intensities were analyzed by Image-Pro Plus 6.0 software and standardized to GAPDH as a loading control. 
Immunofluorescence analysis. Cortical neurons were fixated in 4\% PFA on glass slides overnight, washed with PBS 3 times, and incubated with 3\% BSA in PBS for 10 minutes. Slides were incubated with antiMAP-2 (dilution: 1/300; Abcam, UK) in PBS containing 1\% BSA overnight. Samples were treated with secondary antibodies Alexa Fluor 488 IgG (dilution: 1/500; Abcam, UK) at room temperature for $1 \mathrm{~h}$. Slides were finally washed, mounted in VectaShield medium, observed, and photographed on Zeiss Axio Observer Z1, an inverted fluorescent microscope.

Statistical analysis. Data evaluation was based on the unpaired two-tailed Student's t-test for comparing the difference between two samples and one-way ANOVA for multiple comparisons. All data were presented as mean \pm SEM. The value of $P<0.05$ was considered statistically significant.

\section{Results}

\section{Characterization of exosomes from 293T cells}

Exosomes were isolated from 293T cells with Lamp2b overexpression. Western blot was used to analyze the expression of specific antibodies including $C D 9, C D 43$, and $C D 67$, all presented features of exosomes (as shown in Fig. 1). These results suggested exosomes located in the purified addition which could be adopted in further experiments.

\section{Sirna Pooled Knockdown The Expression Of Axonal Growth Inhibitor}

To determine whether the siRNA could mediate the expression of axonal growth inhibitor, we detected the protein expression of the axonal growth inhibitor. As shown in Fig. 2A, expressions of Troy, LIONGO-1, PTEN, and NgR exhibited a sharp decrease. Diminished expression of the axonal growth inhibitor was indicated by the siRNA pool compared with the control group (Fig. 2B). Further analysis demonstrated that the expression of the axonal growth inhibitor was reduced in cortical neurons after being transfected with the siRNA pool.

\section{Transmigration Of Exo-sirna Pool In Primary Cortical Neurons}

Next, the transfection efficiency of the exo-siRNA pool was investigated in $\mathrm{SCl}$ cortical neurons. As illustrated in Fig. 3, the exo-siRNA pool was efficiently transfected into the cortical neurons which exhibited the possibility of the exo-siRNA pool positioned in the cortical neurons after being treated for $6 \mathrm{~h}$. These data indicated that the exo-siRNA pool was specifically located in primary cortical neurons.

Exo-siRNA pool improved the cell proliferation of SCl primary cortical neurons 
In the vita metastasis environment, cytotoxicity of different siRNA (Troy, Lingo-1, NgR, and PTEN) transfections was determined by MTT assay. As described in Fig. 4, the cell proliferation of cortical neurons was declined remarkably in contrast to that of the $\mathrm{SCl}$ group, and the same results were achieved in the $\mathrm{SCl}+$ exsome group and the $\mathrm{SCl}+$ siRNA group compared with the control group. However, an increase in proliferation was revealed in the $\mathrm{SCl}+$ exo-siRNA pool group. Meanwhile, the expression of Rho revealed no apparent alternation in the $\mathrm{SCl}$ group compared with the control group whereas the Rho expression was decreased in the $\mathrm{SCl}+$ exo-siRNA pool group. These data displayed that the exo-siRNA pool significantly alleviated the cell proliferation and Rho expression in primary cortical neurons induced by SCl.

\section{Discussion}

$\mathrm{SCl}$ is a common disease affecting the nervous system, it also causes the onset of paralysis, paresthesia, and nerve pains.[21] Despite great efforts have been made to improve the functional outcome of patients, $\mathrm{SCl}$ remains an issue to be solved with existing therapeutics measures to date. Lately, biological therapeutics provide an alternative for $\mathrm{SCl}$ patients assisting in the realization of spinal cord regeneration in the future.[22]

As a vital component of lysosomal membranes, Lamp2 is involved in autophagy. Wang has ascertained the treatment for exosomes (containing Lamp2b)/miR-26a elevated cross-sectional areas of skeletal muscles, diminished the upregulation of FOX032/atrogin-1, and TRIM63/MuRF1 and depressed cardiac fibrosis lesions.[23] Moreover, exosomes as a kind of extracellular vesicle have emerged as a promising therapeutic delivery platform due to their specific composition and biological properties, providing the nervous system with effective agents for treating various diseases.[24] A recent study has reported that exosomes from serum-deprived mesenchymal stem cells were utilized to deliver siRNAs to neurons.[25] In line with Wang's discovery, exosomes/TRPP2 siRNA complex is capable of suppressing TRPP2 expression of Fadu cells, meanwhile, low expression of TRPP2 elicits an increase in E-cadherin, but downward expression levels of $\mathrm{N}$-cadherin and vimentin.[26] Reports have revealed that rehabilitation of $\mathrm{SCl}$ rats achieved satisfactory results via injecting intravenously with exosomes (control exosomes, miRNA-29b exosomes) and BMSCs (miR NC, miRNA-29b) to the tail vein.[27] Li has suggested that exosomes derived from miR-544 modified BMSCs exhibited a protective role for the $\mathrm{SCl}$ rat model in improving their functional recovery as well as the promotion of neuronal survival.[28] Besides, injection of miR-133b exosomes produces effects in neuron protection, promotion of the axon regeneration, and enhancement of the damaged locomotor function caused by SCI.[29] In light of the previous results, the delivery of siRNA/miRNA by exosomes is assumed to be an effective therapeutic strategy for neuron diseases. In this study, we engineered an exosome vector containing Lamp2b in HEK293T cells with the results hinted that the transfer of either exosome-mediated miRNAs or siRNAs represented a novel therapeutic approach for the treatment of SCl.

Chronic compression of the $\mathrm{SCl}$ results in loss of the anterior motoneurons, while the precise mechanism on how they got lost remains unclear. Our current study investigated the regeneration disorders in the 
CNS and the findings certified that the presence of axonal growth-inhibiting proteins played a crucial role in $\mathrm{SCl}$. NogoA/NgR as axon growth inhibitory factors and the signaling pathways play a vital role in the repair of nerve damages namely multiple sclerosis. By downregulating NogoA/NgR and RhoA/ROCK signaling pathways, satisfactory results were achieved in the relief and repair of axonal injuries for experimental autoimmune encephalomyelitis.[30] Additionally, NgR microRNA-494 has been attested participated in the proliferation of astrocytes and the synaptic remodeling in the $\mathrm{SCl}$ rat model by regulating the Nogo/NgR signaling pathway.[31] LINGO-1 presents a negative regulation for neural regeneration in the CNS. Once the central nerve was injured, the up-regulation of LINGO-1 was noticed and so did the apoptosis of cells. Huang found that the deletion of LINGO-1 was beneficial in improving the spinal cord micro-environment after $\mathrm{SCl}$ and contributing to cell survival.[32] PTEN has been identified as a prominent intrinsic inhibitor of CNS axon regeneration a decade ago. Intensive explorations have confirmed that the PTEN negatively regulated the PI3K-AKT-mTOR pathways based on diverse models of axon injury and diseases with mechanisms affecting axon regeneration more and more obvious. Whereas the deletion of the PTEN expression promotes the survival of retinal ganglion cells and stimulates long-distance axonal regeneration after optic nerve axotomy.[33] Serving as an essential negative regulator, Troy is ascribed to the development of oligodendrocyte. Moreover, the knockdown of Troy in situ transplantation of OPCs leads to notable demyelination and neurological recovery in rats with SCI.[34] Based on the described results, all of the NgR, LINGO-1, PTEN, and Troy are assured being involved in the axon injury during $\mathrm{SCl}$ which might interact with each other when neurons were damaged. In our study, we constructed a siRNA pool with results indicating that the expressions of axonal growth inhibitors were reduced remarkably with an increase of cell proliferation and alleviation of the Rho expression in primary spinal cord forms of $\mathrm{SCl}$ rats, thereby identifying a promising target mechanism of $\mathrm{SCl}$ by modifying the amount of axonal growth inhibitor.

\section{Conclusion}

In this paper, we performed an investigation to explore the effect of the transfer of exosome-mediated siRNA pool, which specifically decreased the expression of axonal growth inhibitor proteins, and discovered that the target of the siRNA pool was the cortical neurons. Upon the transfection of the exosiRNA pool, both cell proliferation and Rho expression have been confirmed being alleviated. These findings demonstrate that exosomes with Lamp2b loaded siRNA pool exert roles in improving the functional cortical neurons. The effect of axonal growth inhibitor on pathological change of $\mathrm{SCl}$ and cortical neuron damage might be recovered by performing the knockdown of axonal growth inhibitors.

\section{Declarations}

\section{Availability of data and materials}

Not applicable.

\section{Acknowledgement}


Not applicable.

\section{Conflicts of Interest}

The authors declare that they have no competing interests.

\section{Funding Statement}

Not applicable.

\section{Personal Financial Interests}

Not applicable.

\section{References}

1. Duran RC, Yan H, Zheng Y, Huang X, Grill R, Kim DH, Cao Q, Wu JQ. The systematic analysis of coding and long non-coding RNAs in the sub-chronic and chronic stages of spinal cord injury. Sci Rep. 2017,7,41008.

2. Ryu JC, Tooke K, Malley SE, Soulas A, Weiss T, Ganesh N, Saidi N, Daugherty S, Saragovi U, Ikeda Y, et al. Role of proNGF/p75 signaling in bladder dysfunction after spinal cord injury. J Clin Invest. 2018;128(5):,1772-86.

3. Sepe M, Lignitto L, Porpora M, Delle Donne R, Rinaldi L, Belgianni G, Colucci G, Cuomo O, Viggiano D, Scorziello A, et al. Proteolytic control of neurite outgrowth inhibitor NOGO-A by the cAMP/PKA pathway. Proc Natl Acad Sci U S A. 2014;111(44):,15729-34.

4. Lawson C, Vicencio JM, Yellon DM, Davidson SM. Microvesicles and exosomes: new players in metabolic and cardiovascular disease. J Endocrinol. 2016;228(2):,R57-71.

5. Liu Y, Ma Y, Zhang J, Yuan Y, Wang J, Exosomes. A Novel Therapeutic Agent for Cartilage and Bone Tissue Regeneration. Dose Response. 2019;17(4):1559325819892702.

6. Perets N, Hertz S, London M, Offen D. Intranasal administration of exosomes derived from mesenchymal stem cells ameliorates autistic-like behaviors of BTBR mice. Mol Autism. 2018,9,57.

7. Ghoreishy A, Khosravi A, Ghaemmaghami A. Exosomal microRNA and stroke: A review. J Cell Biochem. 2019;120(10):16352-61.

8. Huang S, Ge X, Yu J, Han Z, Yin Z, Li Y, Chen F, Wang H, Zhang J, Lei P. Increased miR-124-3p in microglial exosomes following traumatic brain injury inhibits neuronal inflammation and contributes to neurite outgrowth via their transfer into neurons. FASEB J. 2018;32(1):512-28.

9. Christodoulou A, Santarella-Mellwig R, Santama N, Mattaj IW. Transmembrane protein TMEM170A is a newly discovered regulator of ER and nuclear envelope morphogenesis in human cells. J Cell Sci. 2016;129(8):;1552-65.

10. Liu F, Gao ML, Bai J, Wang YF, Li XQ. Inhibition of neurite outgrowth using commercial myelin associated glycoprotein-Fc in neuro-2a cells. Neural Regen Res. 2018;13(11):,1893-9. 
11. Terzi YK, Oguzkan-Balci S, Anlar B, Erdogan-Bakar E, Ayter S. Learning disability and oligodendrocyte myelin glycoprotein (OMGP) gene in neurofibromatosis type 1. Turk J Pediatr. 2011;53(1):75-8.

12. Wang H, Song G, Chuang H, Chiu C, Abdelmaksoud A, Ye Y, Zhao L. Portrait of glial scar in neurological diseases. Int J Immunopathol Pharmacol. 2018,31,2058738418801406.

13. Ahmed Z, Dent RG, Suggate EL, Barrett LB, Seabright RJ, Berry M, Logan A. Disinhibition of neurotrophin-induced dorsal root ganglion cell neurite outgrowth on CNS myelin by siRNA-mediated knockdown of NgR, p75NTR and Rho-A. Mol Cell Neurosci. 2005;28(3):,509-23.

14. Fu C, Wei Z, Zhang DPTEN. Inhibits Inflammatory Bone Loss in Ligature-Induced Periodontitis via IL1 and TNF-alpha. Biomed Res Int. 2019,2019,6712591.

15. Ma J, Zhang Y, Chen L, Chen Y, Yang Y, Li D, Wang L, Zhang J, Wu Z. Low Expression of Phosphatase and Tensin Homolog and High Expression of Ki-67 as Risk Factors of Prognosis in Cranial Meningiomas. World Neurosurg. 2020,136,e196-e203.

16. Xing HY, Meng EY, Xia YP, Peng H. Effect of retinoic acid on expression of LINGO-1 and neural regeneration after cerebral ischemia. J Huazhong Univ Sci Technolog Med Sci. 2015;35(1):54-7.

17. Agundez JA, Jimenez-Jimenez FJ, Alonso-Navarro H, Garcia-Martin E. The potential of LINGO-1 as a therapeutic target for essential tremor. Expert Opin Ther Targets. 2015;19(8):1139-48.

18. Lee JY, Kim MJ, Li L, Velumian AA, Aui PM, Fehlings MG, Petratos S. Nogo receptor 1 regulates Caspr distribution at axo-glial units in the central nervous system. Sci Rep. 2017;7(1):8958.

19. Naito A, Yoshida H, Nishioka E, Satoh M, Azuma S, Yamamoto T, Nishikawa S, Inoue J. TRAF6deficient mice display hypohidrotic ectodermal dysplasia. Proc Natl Acad Sci U S A. 2002;99(13):,8766-71.

20. Pacifici M, Peruzzi F. Isolation and culture of rat embryonic neural cells: a quick protocol. $J$ Vis Exp. 2012(63),e3965.

21. David G, Mohammadi S, Martin AR, Cohen-Adad J, Weiskopf N, Thompson A, Freund P. Traumatic and nontraumatic spinal cord injury: pathological insights from neuroimaging. Nat Rev Neurol. 2019;15(12):,718-31.

22. Zhou P, Guan J, Xu P, Zhao J, Zhang C, Zhang B, Mao Y, Cui W. Cell Therapeutic Strategies for Spinal Cord Injury. Adv Wound Care (New Rochelle). 2019;8(11):585-605.

23. Wang B, Zhang A, Wang H, Klein JD, Tan L, Wang ZM, Du J, Naqvi N, Liu BC, Wang XH. miR-26a Limits Muscle Wasting and Cardiac Fibrosis through Exosome-Mediated microRNA Transfer in Chronic Kidney Disease. Theranostics. 2019;9(7):1864-77.

24. Valentino A, Reclusa P, Sirera R, Giallombardo M, Camps C, Pauwels P, Crispi S, Rolfo C. Exosomal microRNAs in liquid biopsies: future biomarkers for prostate cancer. Clin Transl Oncol. 2017;19(6):651-7.

25. Haraszti RA, Miller R, Dubuke ML, Rockwell HE, Coles AH, Sapp E, Didiot MC, Echeverria D, Stoppato $M$, Sere YY, et al. Serum Deprivation of Mesenchymal Stem Cells Improves Exosome Activity and Alters Lipid and Protein Composition. iScience. 2019,16,230-241. 
26. Wang C, Chen L, Huang Y, Li K, Jinye A, Fan T, Zhao R, Xia X, Shen B, Du J, et al. Exosome-delivered TRPP2 siRNA inhibits the epithelial-mesenchymal transition of FaDu cells. Oncol Lett. 2019;17(2):,1953-61.

27. Yu T, Zhao C, Hou S, Zhou W, Wang B, Chen Y. Exosomes secreted from miRNA-29b-modified mesenchymal stem cells repaired spinal cord injury in rats. Braz J Med Biol Res. 2019;52(12):,e8735.

28. Li C, Li X, Zhao B, Wang C. Exosomes derived from miR-544-modified mesenchymal stem cells promote recovery after spinal cord injury. Arch Physiol Biochem. 2020,1-7.

29. Li D, Zhang P, Yao X, Li H, Shen H, Li X, Wu J, Lu X. Exosomes Derived From miR-133b-Modified Mesenchymal Stem Cells Promote Recovery After Spinal Cord Injury. Front Neurosci. 2018,12,845.

30. Fang L, Wang Y, Zheng Q, Yang T, Zhao P, Zhao H, Zhang Q, Zhao Y, Qi F, Li K, et al. Effects of Bu Shen $\mathrm{Yi}$ sui capsule on NogoA/NgR and its signaling pathways RhoA/ROCK in mice with experimental autoimmune encephalomyelitis. BMC Complement Altern Med. 2017;17(1):346.

31. Lim L, Balakrishnan A, Huskey N, Jones KD, Jodari M, Ng R, Song G, Riordan J, Anderton B, Cheung ST, et al. MicroRNA-494 within an oncogenic microRNA megacluster regulates $\mathrm{G} 1 / \mathrm{S}$ transition in liver tumorigenesis through suppression of mutated in colorectal cancer. Hepatology. 2014;59(1):202-15.

32. Huang LJ, Li G, Ding Y, Sun JH, Wu TT, Zhao W, Zeng YS. LINGO-1 deficiency promotes nerve regeneration through reduction of cell apoptosis, inflammation, and glial scar after spinal cord injury in mice. Exp Neurol. 2019,320,112965.

33. Huang ZR, Chen HY, Hu ZZ, Xie P, Liu QH. PTEN knockdown with the Y444F mutant AAV2 vector promotes axonal regeneration in the adult optic nerve. Neural Regen Res. 2018;13(1):135-44.

34. Sun L, Liu S, Sun Q, Li Z, Xu F, Hou C, Harada T, Chu M, Xu K, Feng X, et al. Inhibition of TROY promotes OPC differentiation and increases therapeutic efficacy of OPC graft for spinal cord injury. Stem Cells Dev. 2014;23(17):,2104-18.

\section{Figures}




\section{CD9}

\section{CD47}

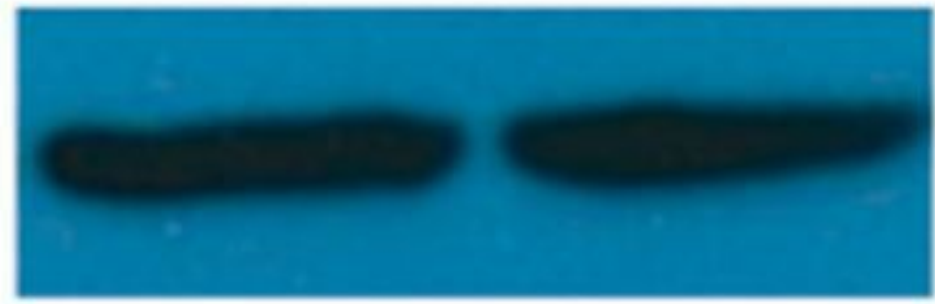

\section{CD63}
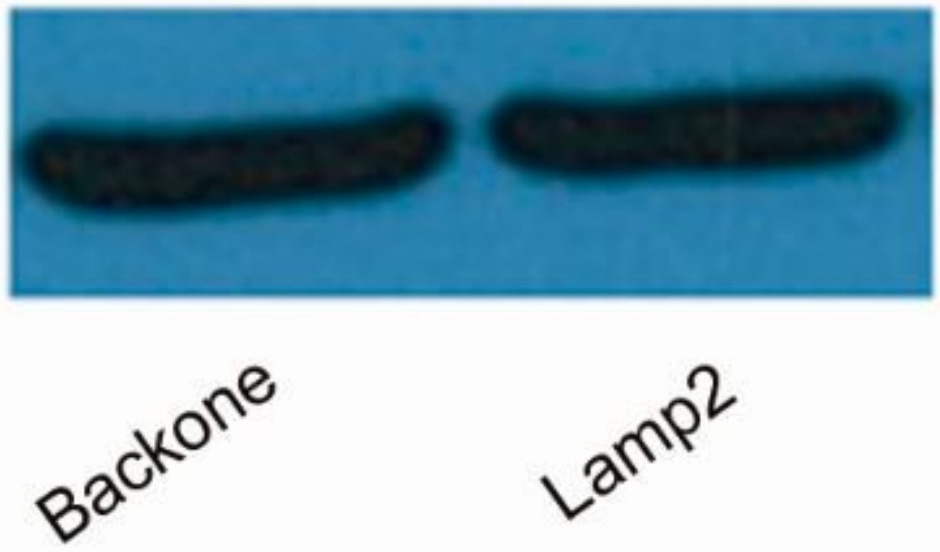

\section{Figure 1}

Preparation of Lamp2b overexpression HEK293T cell-derived exosomes. Exosomes were characterized by a specific marker by using western blot (positive marker: CD9, CD47, and CD63).

A
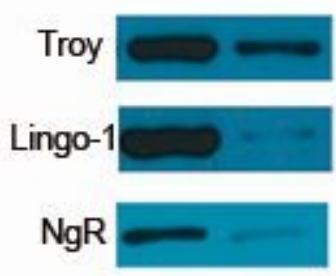

\section{PTEN}

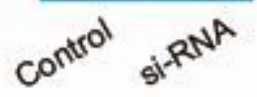

B

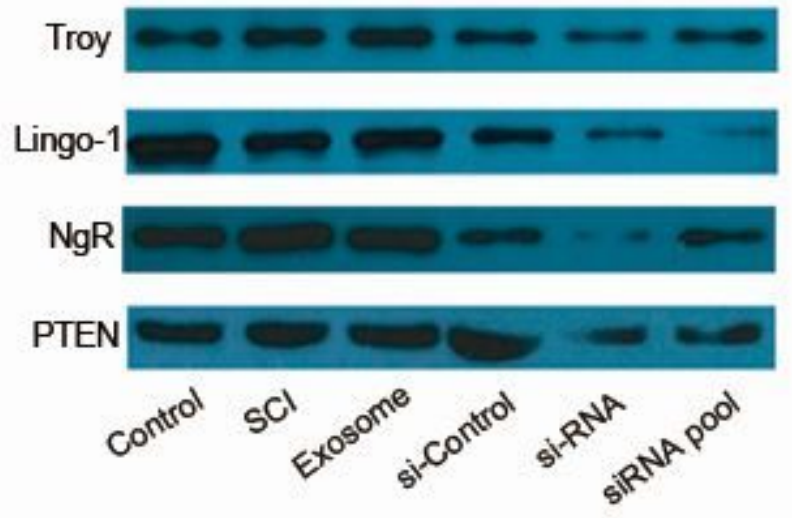

Page 12/14 
Figure 2

Expression of axonal growth inhibitor was down-regulated by siRNA transfection. (A) Western blot analysis of Troy, LINGO-1, NgR, and PTEN after transfected with siRNA. (B) Western blot was used to analyze the expression of Troy, LINGO-1, NgR, and PTEN in cortical neurons after transfected with siRNA. A
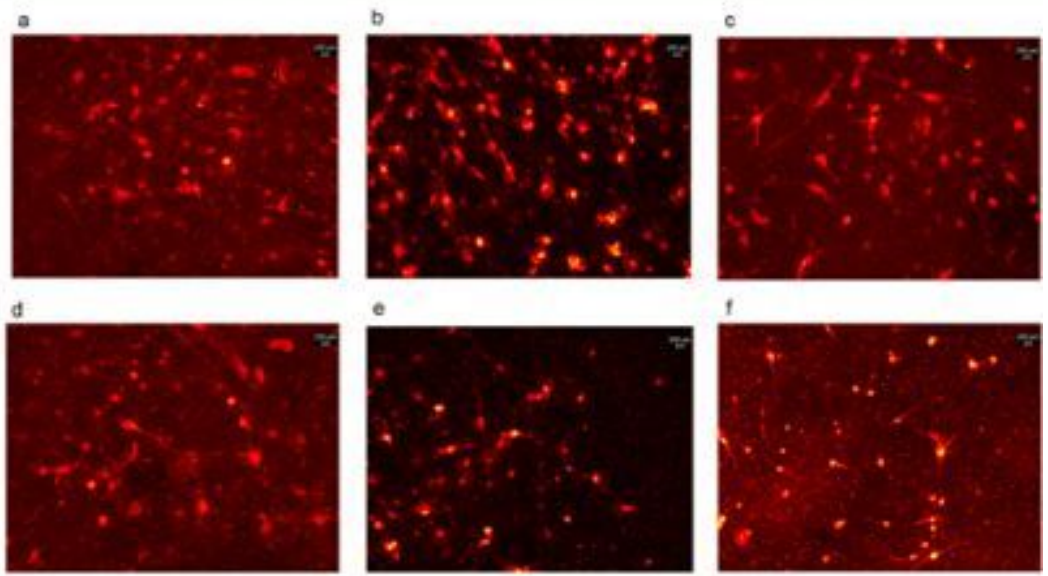

9
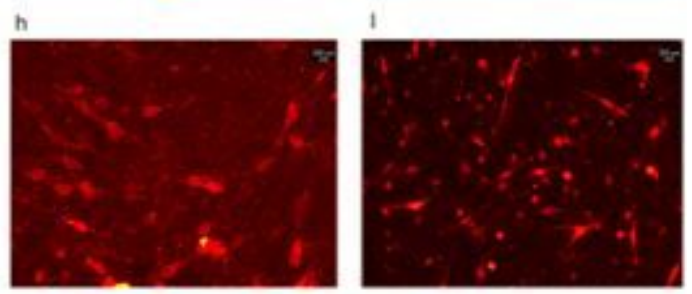

B

SiRNA pool

MAP-2

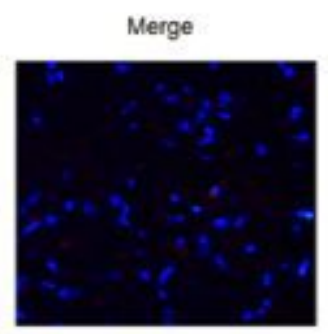

Control
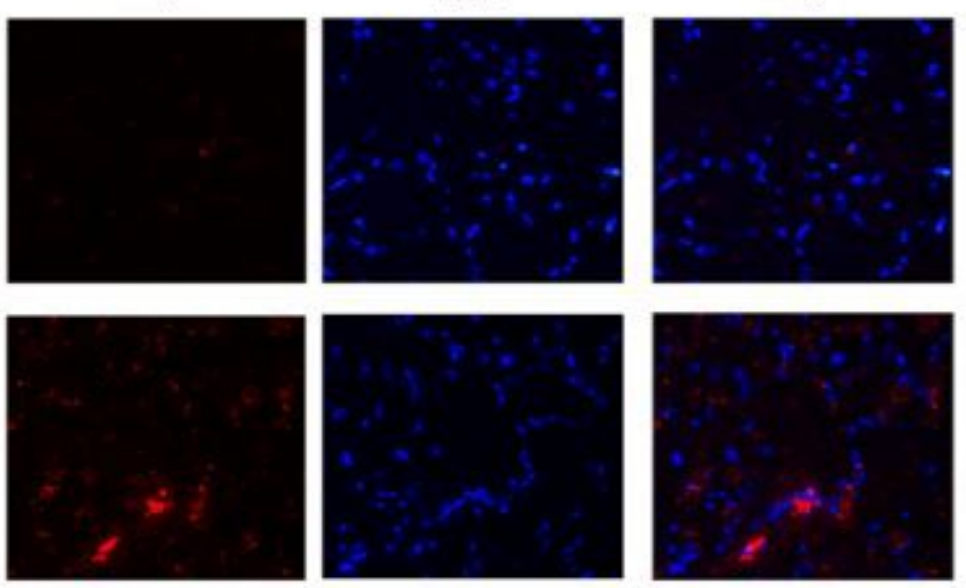

\section{Figure 3}

The transmigration of exo-siRNA pool in primary cortical neurons. (A) The transfection efficiency of siRNA in primary cortical neurons. a: Control; b: SCl; c: SCl+exosome; d: SCl+siCon; e: SCl+exosome+siTroy; f: SCl+exosome+siLINGO-1; g: SCl+exosome+siNgR; h: SCl+exosome+siPTEN; i: SCl+exosome+siRNA pool. (B): Transmigration of exo-siRNA pool in primary cortical neurons. 
A

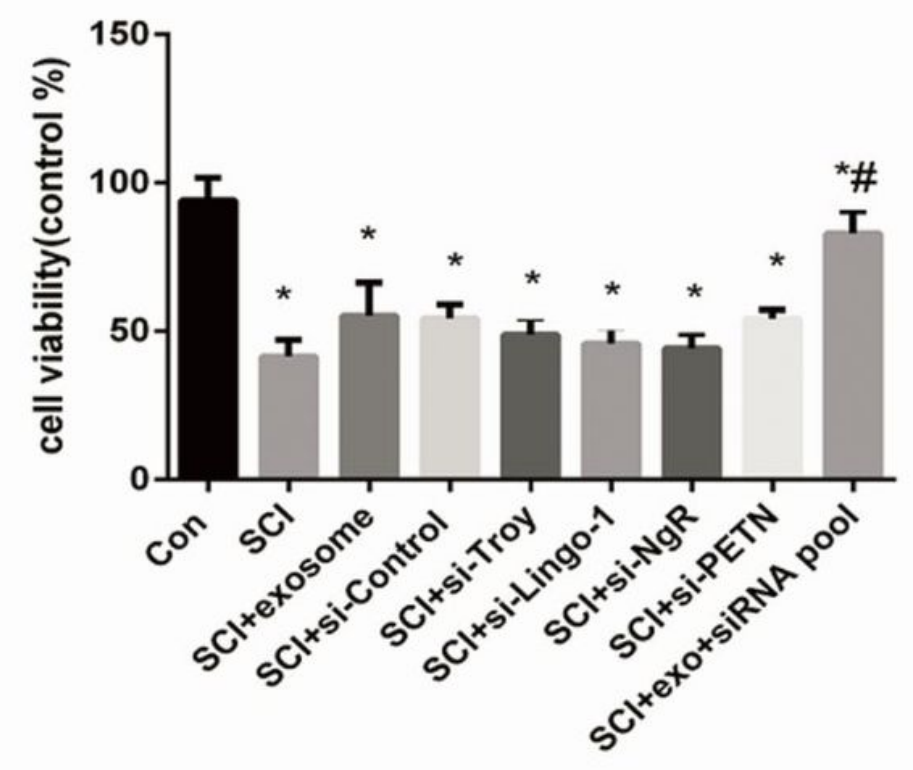

B

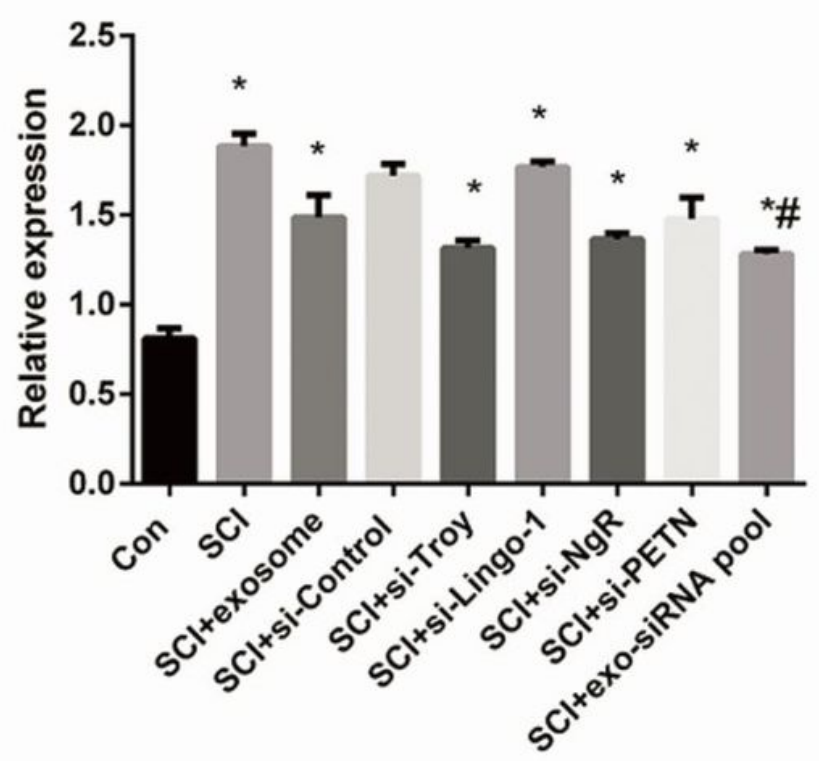

Figure 4

Exo-siRNA pool alleviated the neuron damage induced by SCI. A: Cell proliferation of cortical neurons. B: The expression of Rho in cortical neurons. *:p<0.05, compared with control; \#: $p<0.05$, compared with SCI group. 